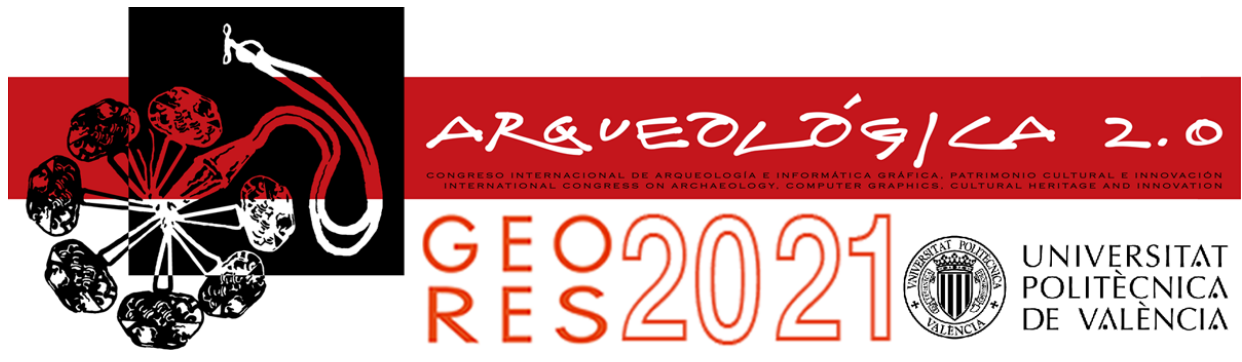

Proceedings of the joint international event $9^{\text {th }}$ ARQUEOLÓGICA

$2.0 \& 3^{\text {rd }}$ GEORES

Valencia (Spain).

26-28 April 2021

\title{
INTEGRATION OF COMPLEX 3D MODELS INTO VR ENVIRONMENTS: CASE STUDIES FROM ARCHAEOLOGY
}

\author{
Julia Gorkovchuka, ${ }^{a}$, Denys Gorkovchuka,b, Thomas Luhmann ${ }^{c}$ \\ ${ }^{a}$ Kiev National University for Construction and Architecture, Kyiv, Ukraine. gorkovchukjulia@gmail.com \\ b SPM3D LLC, Kyiv, Ukraine. denys.gorkovchuk@spm3d.com \\ ${ }^{\mathrm{c}}$ Institute for Applied Photogrammetry and Geoinformatics, Jade University of Applied Sciences, 26121 Oldenburg, Germany. \\ luhmann@jade-hs.de
}

\begin{abstract}
:
Recently, virtual reality technologies are increasingly being introduced into our lives. The focus of their use is shifting from the entertainment industry to design, healthcare, tourism, architecture, education and more. The advantages of virtual reality technology are especially noticeable in the field of archaeology, as many historical objects have not survived to our time, and their appearance can be reproduced only on the basis of historical sources and archaeological excavations. Most platforms for implementing virtual reality programs are based on game engines that can provide the required level of performance for VR. Such platforms show very good results for architectural objects, which often have many similar elements of simple shapes. Integrating complex objects with unique shapes is usually a problem. In this article, we consider the use of photogrammetric methods to create 3D models of historical objects and the aspects of their integration into a virtual environment based on a game engine. Specifically, aspects such as object resolution and suitable level of detail are discussed. As a case study, such a virtual environment was created for the ancient Trypillia settlement in the territory of Ukraine.
\end{abstract}

Keywords: archaeology, photogrammetry, 3d modelling, historical reconstruction, virtual reality, immersive technologies

\section{Introduction}

\subsection{Historical reconstruction in virtual reality}

To cover the history of mankind on the basis of artefacts that lie at the depth of cultural and geological layers can be referred to as the main task of the ancient science of archaeology which today, due to scientific progress, has received state-of-the-art tools and research methods (Terpylovsky, 2008).

The purpose of the reconstruction of historical and cultural heritage sites is, first of all, their preservation and rational use, the formation of protected areas, historical areas of settlements and historical environment. It is important to create investment-attractive conditions for the rehabilitation, reconstruction and use of buildings and structures that have a certain historical, cultural or architectural and artistic value as carriers of the traditional nature of the environment.

This paper deals with historical reconstruction as a method of restoring the appearance and construction of an object, based on its preserved fragments, remains and available historical information. The solution to this problem is realized by immersive technologies: a term that defines the technology of human interaction with space, information, content in the real and virtual worlds. The modelling of the historical environment by these technologies has a number of advantages:

- clarity: in the virtual space you can consider in detail any process or object;

- accuracy: not limited by the technical capabilities of the physical embodiment of objects;

- maximum involvement: the ability to fully control and change the scenario of events;

- safety: no risks to humans and the environment;

- cost: no costs for logistics, materials, etc.

\subsection{The current state of use of immersive technologies in virtual reality}

Nowadays, immersive technologies are used in almost all areas of activity from school education to artificial intelligence. Considering different levels of user involvement, they can be classified into: 
- VR (virtual reality): require a special headset which immerse a person in a fictional or recreated environment;

- $\quad$ AR (augmented reality): literally supplements the existing environment with virtual images, animations, visual effects;

- $\quad \mathrm{RR}$ (real reality): a real-world which is not virtual or augmented;

- MR (mixed reality): similar in meaning to augmented, but instead of "embedding" images or text over the reality, it creates virtual objects or elements, which co-exist and interact with real-world objects;

- $\quad$ XR (cross reality): a combination of VR, AR and $M R$, which allows to bring digital objects into the physical world and bring physical world objects into the digital world.

Immersive technologies are often used to present objects with difficult or dangerous access to the public. As example, detailed models created with laser scanning are integrated into VR environment which allows to walk inside of the caves (Büyüksalih, et al., 2020).

One of the options for improving the quality of VR models is the integration of panoramic images into the virtual $3 D$ model. It allows to significantly improve the level of details for 3D models (Kersten \& Walmsley, 2020a).

$3 \mathrm{D}$ models of historical objects can be used in learning processes for knowledge transfer in virtual environments (Kersten, Trau, \& Tschirschwitz, 2020).

Virtual reality is also being applied for the reconstruction of ancient cities. Typically, traditional surveying methods (laser scanning or photogrammetry) are used to build 3D model of the city territory and ancient maps are used as a basis for 3D reconstruction (Kersten \& Walmsley, 2020b).

It is worth mentioning that currently immersive technologies in archaeological research are illustrative and concern with the reproduction of artefacts in virtual reality. For example, modelling archaeological findings in 3D and publishing them on online platforms (e.g. Sketchfab) (Historical Truth, 2019). No additional software or hardware is required to view such models.

The Lithodomos VR project gives a nice example for the implementation of full virtual reality for archaeology is the development of Spanish archaeologist Lith - a special application for virtual reality devices that will bring users to the ancient world of reconstructed historical sites, including Ancient Greek Olympia, Jerusalem and others (Ancient Worlds, 2020).

Several projects in digitizing of historical objects and creating 3D models were conducted in Ukraine. They were aimed at the development of AR touristic guidebooks and the creation of physical scaled copies of these objects to interact with blind people (Rudyi, 2019).

One of the most progressive uses of virtual reality for archaeological purposes is creating fully virtual archaeological sites with the integration of historical maps, architectural plans and other information in one place to organize teamwork of researchers within one complete virtual environment (Paradis, et al., 2019).

\subsection{Objectives}

The aim of the following project is the integration of complex 3D models into a virtual environment for realtime visualization of an ancient city.

\section{Modelling approach}

\subsection{Object description}

The state historical and cultural reserve "Trypillya culture" is currently the only Ukrainian museum institution of one archaeological culture, which includes 11 Trypillia settlements located in the Cherkasy region, Ukraine. Most famous are "Talyanki" (Fig. 1), "Maidanets", "Dobrovody", "Kosenivka": the largest Eneolithic settlements in the world in the IV millennium BC.

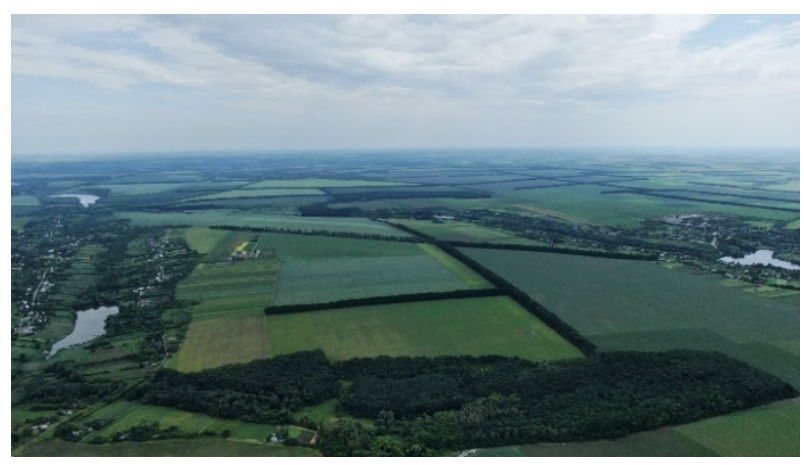

Figure 1: Overview of the "Talyanki" settlement territory.

The Talyanki settlement was chosen as a modelling object for this work. It comprises an area of 450 hectares and can be dated back to the first half of the IV millennium $\mathrm{BC}$.

General dimensions and outlines of the settlement have been established according to extracted data from aerial imagery of 1966. Later in 1983-1986 a geomagnetic survey showed that the settlement consisted of about 1,500 to 2,800 buildings located in concentric circles. In 2011-2012 the geomagnetic survey was repeated with modern equipment which made it possible to determine not only the exact location of buildings, but also their size.

Around 30 objects in that area were studied by archaeological excavations, mostly addressed to the remains of burned houses. As a part of experimental archaeological studies, two of such buildings were reconstructed using old technologies in the territory of "Trypillya culture" museum in Lehedzyne, Cherkasy region, Ukraine.

The Northern part of the settlement (200 hectares) was chosen for the modelling in this research project.

\subsection{Data acquisition and modelling}

Data collection was conducted with photogrammetric methods.

As a first step, the territory of the settlement was surveyed with a DJI Mavic 2 Pro UAV. 350 images with a ground resolution of around $8 \mathrm{~cm}$ were taken (Fig. 2). 


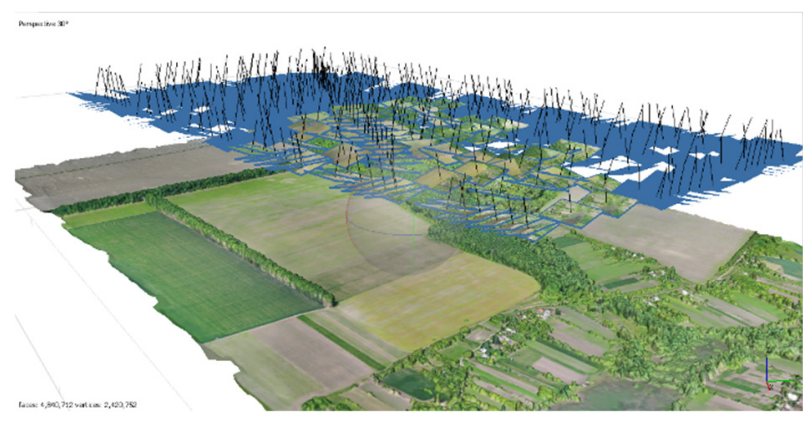

Figure 2: UAV camera positions and 3D model of the settlement territory.

An orthophoto, a DSM and a DEM of the territory were generated (Fig. 3). They were used as a basis for the integration of all further data.

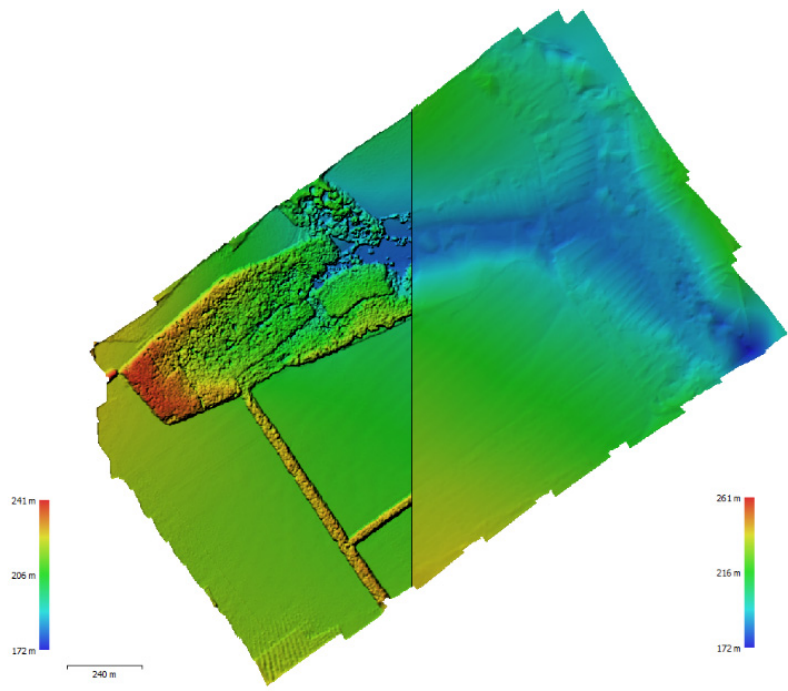

Figure 3: Left: DSM; Right: DEM of the territory.

The results of geomagnetic survey, conducted in 2011 2012, were superimposed to the created model of the territory (Fig. 4). Black spots represent the remains of burned clay which was used as floor for Trypillya houses. They have a clear rectangular shape that allows to determine the size of the building. Distribution of these buildings allows to discover the locations of streets in an ancient city.

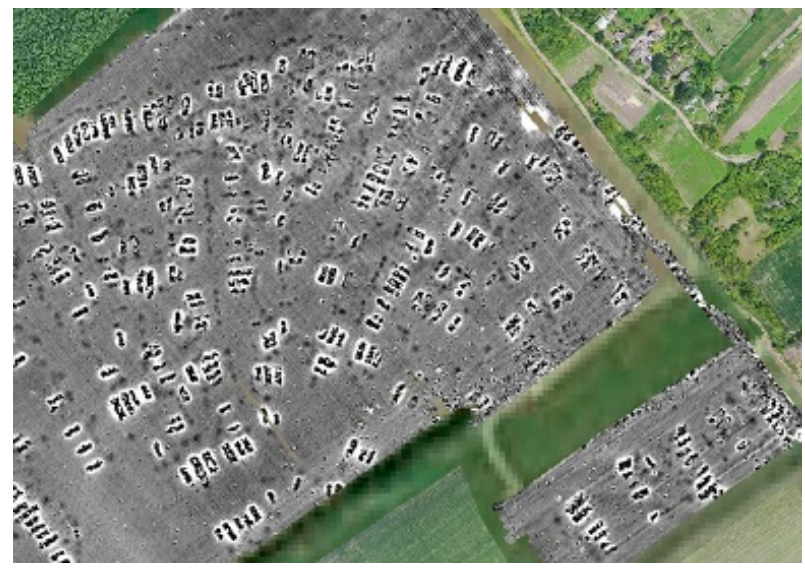

Figure 4: Orthophoto of the territory with superimposed geomagnetic survey.
The second step of data acquisition was the capturing of Trypillya buildings which were reconstructed in the territory of the museum. The buildings have $6 \times 13$ meters footprint and $8 \mathrm{~m}$ height. Again, photogrammetric methods have been applied for this purpose. The same DJI Mavic 2 Pro was used to capture the roof part and a Nikon D3200 DSLR camera was used for taking terrestrial images. 1100 images were captured in total (Fig. 5).

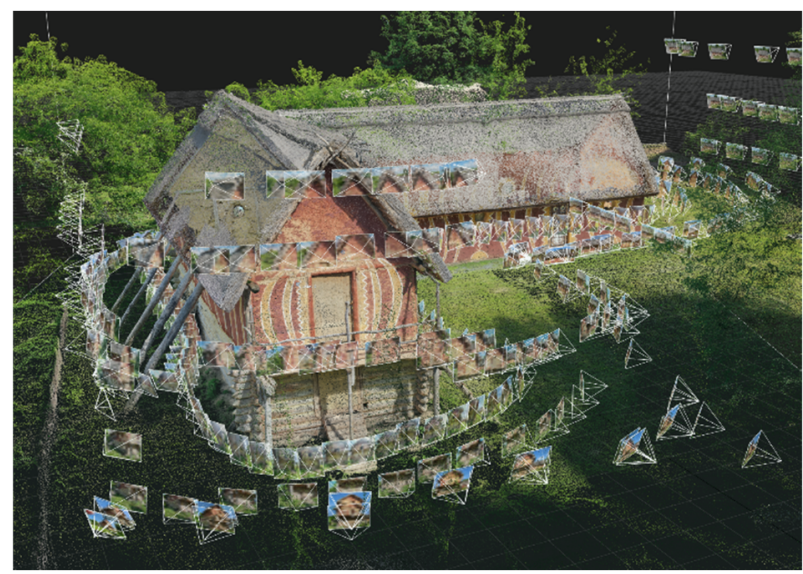

Figure 5: Camera positions and 3D model of the reconstructed Trypillya buildings.

Processing was conducted in RealityCapture software. Due to high overlap, good textures, absence of shiny and reflective surfaces, all of the photos were aligned with a mean reprojection error of 0.6 pixels. Several reference distances were measured for scaling the model and accuracy estimation. The maximum distance error was 10 $\mathrm{mm}$. The cleaned reconstructed mesh model of two buildings consists of about 45 million polygons.

\subsection{Modelling for VR}

Traditionally models generated by photogrammetric methods have millions of polygons and require very powerful workstations and specific software. High quality rendering of such models is usually not possible in real time.

Virtual reality applications require very fast system response (not lower than 60 frames per second) in order to make the interaction with the user comfortable and not stressful. For this purpose, photogrammetric models need to be modified.

The DTM model has been subsampled to $1.0 \mathrm{~m}$ resolution which didn't affect the accuracy significantly, as most of the territory is flat.

To get acceptable performance for building model visualization it has to be simplified to around $50 \mathrm{k}$ polygons. Such significant reduction of polygons $(0.001 \%$ from the full data) results in a loss of quality. To preserve realistic visualization of such simplified model, the simplification process has to be done adaptively - more points remain in complex areas, whereas simple areas are treated in a higher grade of reduction. After reducing the number of polygons the new simplified model is texturized with the original images, hence small elements remain as texture. There is no noticeable difference between texturized models for 20 million and 50k polygon models (Figs. 6 and 7) 


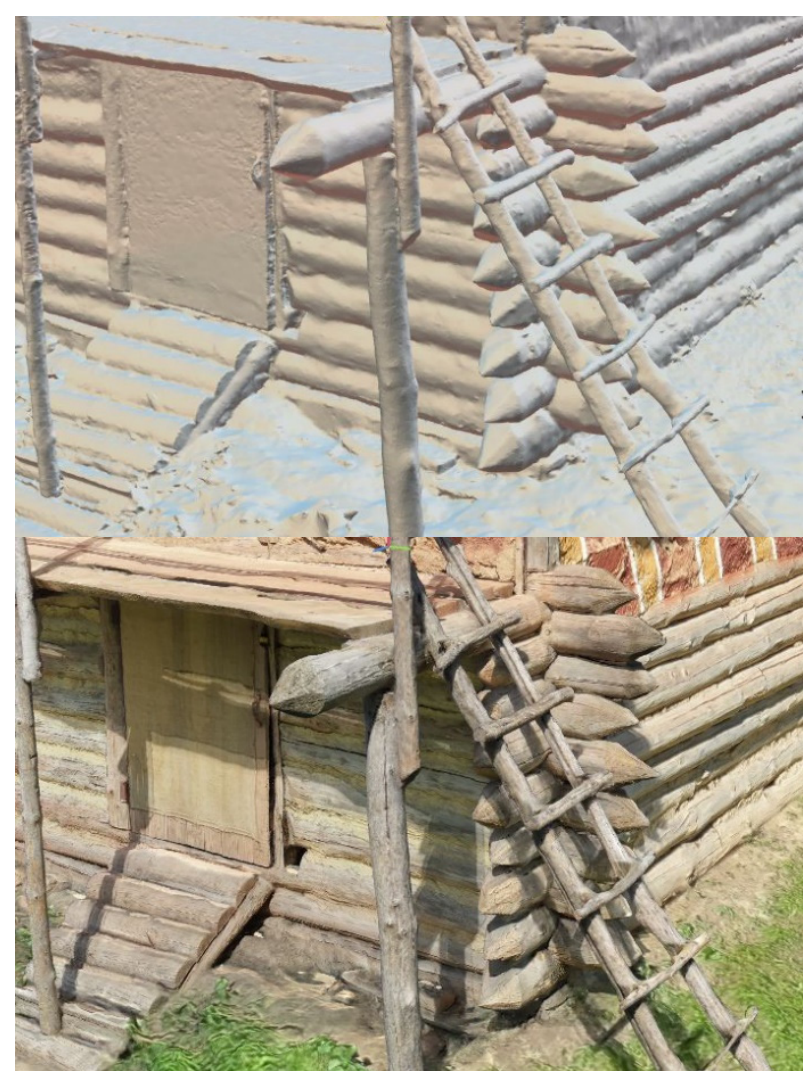

Figure 6: Top: 20 million polygon model without textures; bottom: same model with textures.

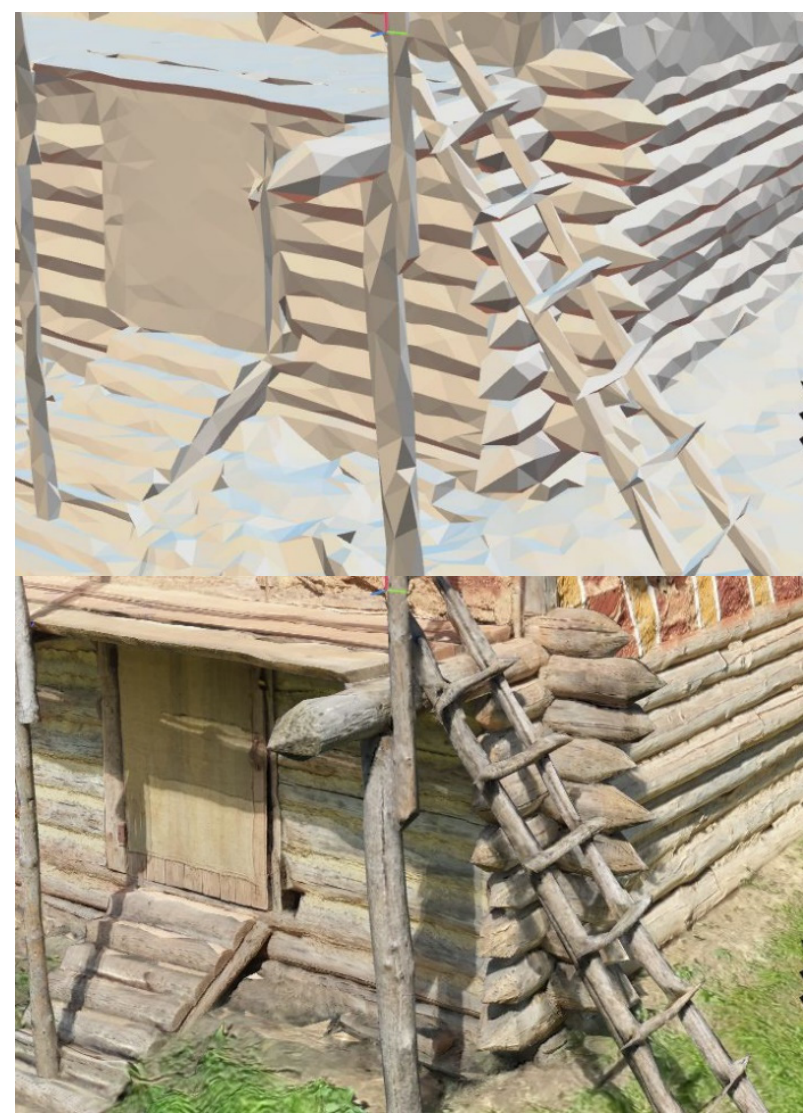

Figure 7: Top: 50k polygon model without textures; bottom: same model with textures.

\section{Virtual reality creation}

\subsection{Description of chosen VR platform}

The reconstruction of settlement in VR was done in Twinmotion software. It is a real-time 3D-immersion software designed mostly for architectural visualizations. It is based on the Unreal Engine, which is used typically for game development.

Twinmotion has a library of different environment elements (trees, grass, stones etc.) and realistic materials which can be used for photorealistic reconstruction.

It also supports the import of digital terrain models and textured 3D models of objects.

\subsection{Data import and modelling aspects}

Twinmotion (same as most game engines) supports only importing several "game-ready" 3D formats.

A terrain model is the base for the virtual reconstruction of the city and has to be implemented at the first stage. The terrain model is critical for correct navigation within the virtual environment (to avoid falling under or flying above the ground) and need to be prepared in a special format, which guarantees a correct collision generation. It is represented as a $2.5 \mathrm{D}$ terrain model, similar to GIS raster formats. However, traditional formats (e.g. GeoTIFF) are not supported directly.

The generated terrain model was exported as GeoTIFF with $1 \mathrm{~m}$ resolution and converted to a grayscale 16-bit PNG image using GRASS GIS software. This allows importing the DEM into the game engine.

Importing of orthophotos is also not supported directly. The results of the geomagnetic survey given as an orthophoto were projected onto the DEM model and imported as a 3D textured model. It allows integrating building locations into Twinmotion. Subsequently, after the modelling of buildings is finished, this model will be removed.

3D textured models of buildings obtained from photogrammetry can be converted to OBJ-format and easily imported into the software.

The next stage is "building" a city with imported models and existing libraries. Building locations and orientations are obtained from geomagnetic survey. Other elements (roads, natural objects) are modelled based on archaeological assumptions.

\subsection{Results}

The use of game engines for generation of virtual environments allows to create a realistic reconstruction of historical objects with very high level of detail. The combination of optimized textured photogrammetric models with highly detailed environment elements (grass, bushes, trees) and visual effects (shadows, sun and cloud movement) an gives impressive photorealistic visualisation in real time (Fig. 8). 


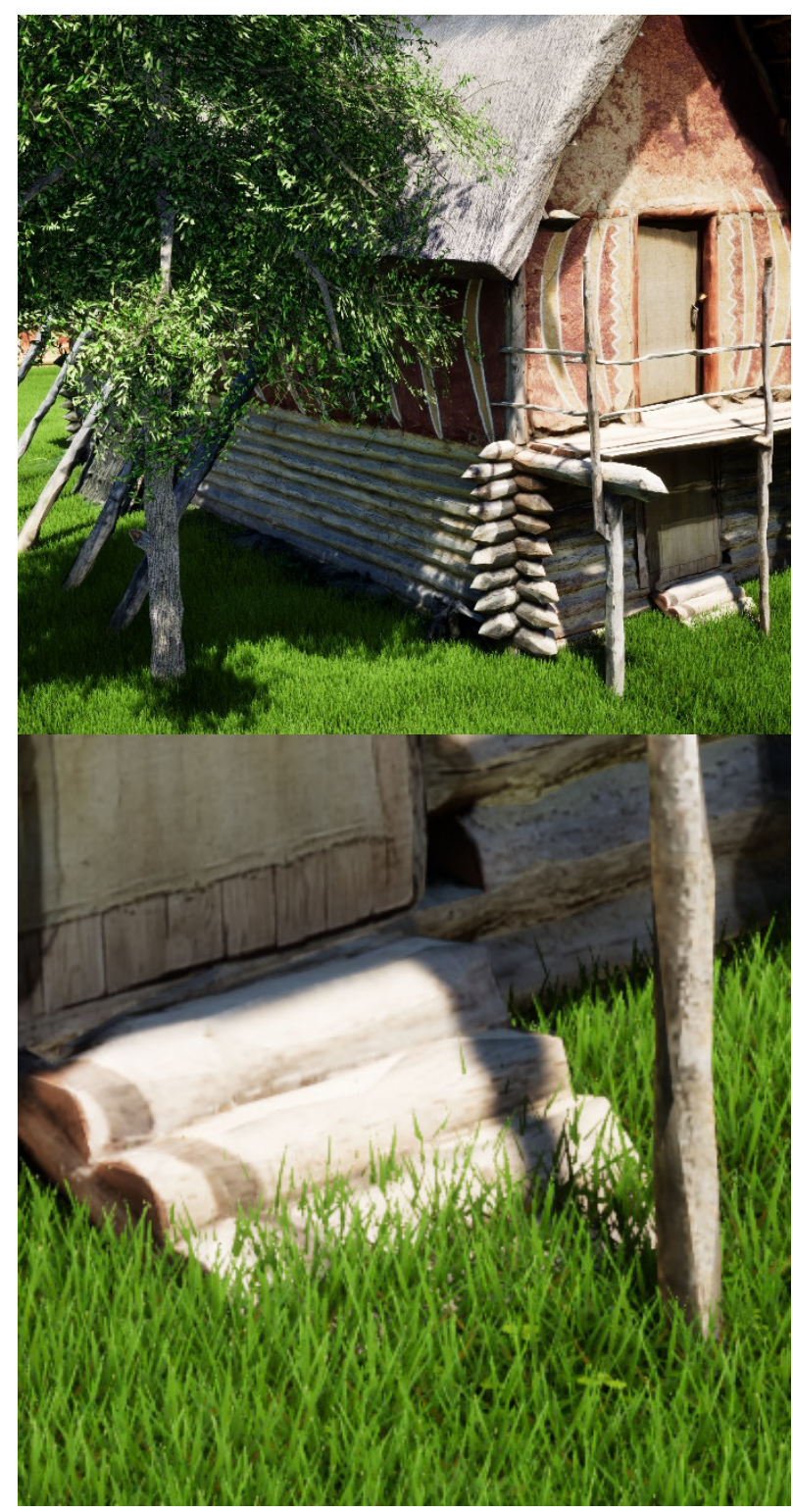

Figure 8: Level of detail for real-time photorealistic visualization in Twinmotion.

Photogrammetry enables the creation of realistic models for such virtual environments. The ability to render them in real-time provides an immersive experience to the user.

The whole project can be exported in different formats such as image renderings, 360-degree and 3D videos or as a standalone VR application, where the user is able to walk and overview the virtual environment by himself.

The most important advantage of using a "game engine approach" in historical virtualization is the simplified workflow for the user, who does not require a big team of different specialists (visual effect artists, modellers) for creating realistic renderings.

However, there are still some difficulties with the direct implementation of geodetic survey results (3D models, DEMs, geomagnetic surveys etc.) into game engine environments:

- 3D model modifications needed;

- no support of common GIS-formats:

- $\quad$ problems with large coordinates.
Another problem arises for the modelling of large territories. To maintain the high speed of visualization, game engines reduce the level of detail when many objects are present in the scene. Figure 9 illustrates the visualization of a complete city with reduced quality: small objects (e.g. grass, bushes and some trees) are not rendered, some objects have missing shadows, textures are simplified.

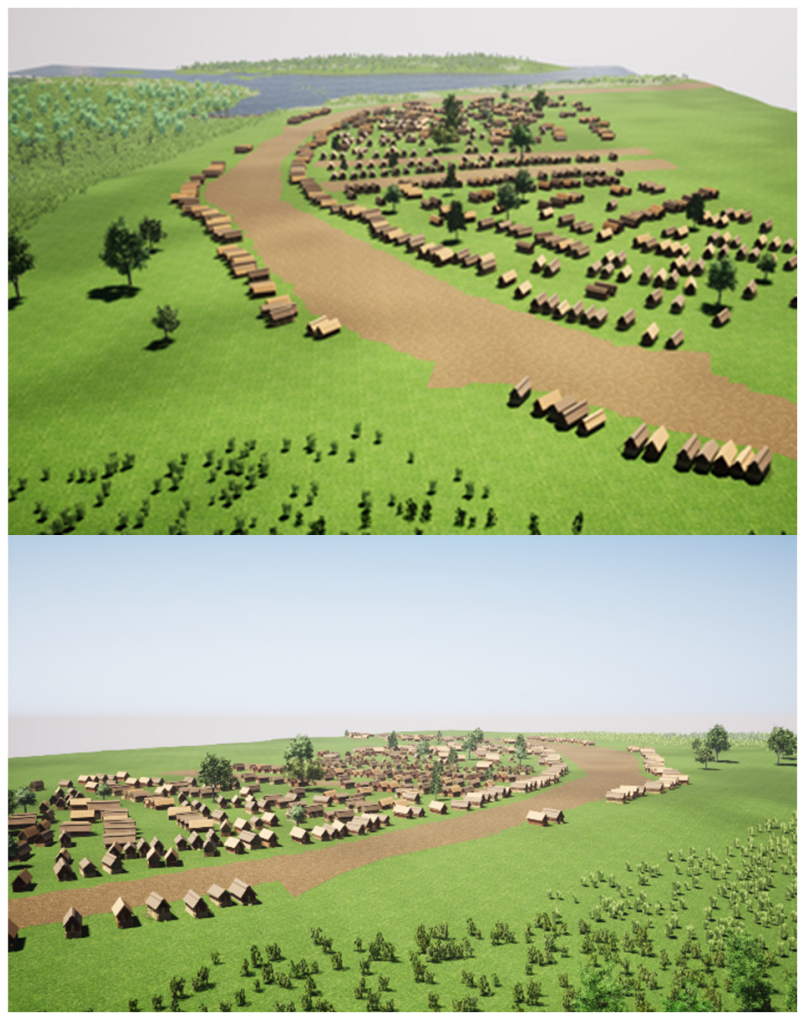

Figure 9: Reduced level of detail for complete settlement visualization.

This is due to specific properties of Twinmotion software, which is not designated for modelling of large spaces. One of the possible solutions of this problem is creating models with several levels of detail (LOD), which are similar to raster-pyramids in GIS. In this case, when the distance from the object to the camera is increasing, a more detailed model will be removed from memory and replaced with a simpler one. This approach allows to use very detailed (up to millimetre resolution) 3D models in game engine environment.

\section{Discussion}

Virtual reality technologies are becoming widespread in many areas and historical reconstruction is one of the most suitable for it. Modern game engines allow you to reproduce in real time a virtual environment of photorealistic quality. Photogrammetry provides an efficient workflow for fast creating of highly detailed 3D models for integrating into such virtual environments.

\section{Conclusion}

Summarizing the results of the study we can draw the following conclusions: 
- $\quad$ photogrammetric methods allow to reconstruct 3D models of unique historic buildings with a high level of detail and low cost;

- modern game engines have proven themselves as a good basis for integrating architectural models into a virtual reality environment, however, integrating models of complex objects with unique shapes into the game engine environment requires additional pre-processing of such models.

- The scope of virtual reconstruction significantly affects the quality of visualization and requires additional configuration of the virtual environment to display objects with different levels of detail.

\section{Further research}

Further research of this project is designated to advanced 3D modelling approaches that allow for the generation of "game-ready" models, with better-optimized textures and LODs. Such models help to avoid simplification problems and allow to render larger areas without a loss of quality.

Another aspect of further research is the use of the "raw" game engine environment, which allows to configure much more parameters and generate results for different platforms, including VR headsets.

\section{References}

Ancient Worlds. (2020). Ancient World - LithodomosVR. Retrieved March 15, 2021, from https://www.lithodomosvr.com/ancient-world/

Büyüksalih, G., Kan, T., Enç, G. Ö., Müge, M., Isın, L., \& Kersten, T. P. (2020). Preserving the Knowledge of the Past Through Virtual Visits: From 3D Laser Scanning to Virtual Reality Visualisation at the Istanbul Çatalca İnceğiz Caves. Journal of Photogrammetry, Remote Sensing and Geoinformation Science volume, 133-146. https://doi.org/10.1007/s41064-020-00091-3

Historical Truth. (2019). 3D-archeology: what the findings of Lviv archaeologists look like. Retrieved March 15, 2021, from https://www.istpravda.com.ua/short/2019/08/2/156028/

Kersten, T. P., Trau, D., \& Tschirschwitz, F. (2020). The four-masted barque peking in virtual reality as a new form of knowledge transfer. ISPRS Ann. Photogramm. Remote Sens. Spatial Inf. Sci., V-4-2020, $155-162$. https://doi.org/10.5194/isprs-annals-V-4-2020-155-2020

Kersten, T., \& Walmsley, A. (2020a). The Imperial Cathedral in Königslutter (Germany) as an Immersive Experience in Virtual Reality with Integrated 360。 Panoramic Photography. Applied Sciences, $10,1517$. https://doi.org/10.3390/app10041517

Kersten, T. P., \& Walmsley, A. P. (2020b). Entwicklung einer interaktiven, immersiven Virtual-RealityApplikation des historischen Stadtmodells Stade 1620. Conference: Publikationen der Deutschen Gesellschaft für Photogrammetrie, Fernerkundung und Geoinformation e.V., Band 29, Tagungsband der 40. Wissenschaftlich-Technischen Jahrestagung der DGPF, 4.-6. März 2020 in Stuttgart, pp. 99-113.

Paradis, M. A., Nicolas, T., Gaugne, R., Barreau, J. B., Auger, R., \& Gouranton, V. (2019). Making virtual archeology great again (without scientific compromise). Int. Arch. Photogramm. Remote Sens. Spatial Inf. Sci., XLII-2/W15,, 879-886. https://doi.org/10.5194/isprs-archives-XLII-2-W15-879-2019

Rudyi, V. (2019). How IT helps preserve and restore architectural landmarks. Retrieved March 15, 2021, from https://nachasi.com/2019/04/16/technologies-architecture/

Terpylovsky, R. (2008). Archeology. Special historical disciplines. Kyiv, Ukraine: Lybid. 